\title{
Potassium Levels, Sources and Time of Application on Nutrient Uptake and Nutrient Use Efficiency of Onion (Allium cepa L.)
}

\author{
B. R. Kumara ${ }^{1 *}$, C. P. Mansur ${ }^{1}$, Shankar Meti ${ }^{1}$, S. L. Jagadeesh ${ }^{1}$, Girish Chander ${ }^{2}$, \\ S. P. Wani ${ }^{2}$, R. K. Mesta ${ }^{1}$, D. Satish ${ }^{1}$, T. B. Allolli ${ }^{1}$ and Sanjeev Reddy ${ }^{1}$
}

${ }^{1}$ Department of Horticulture, College of Horticulture, UHS, Bagalkot, Karnataka, India

${ }^{2}$ ICRISAT-International Crops Research Institute for Semi-Arid Tropics, Patancheru, Telangana, India

*Corresponding author

\section{A B S T R A C T}

The present study on potassium levels, sources and time of application on nutrient uptake and nutrient use efficiency of onion var. ArkaKalyan was carried out at the College of Horticulture, Bagalkot, Karnataka during Kharif season of 2015 and 2016. The uptake of nitrogen, phosphorus, potassium and zinc recorded significantly in 200 per cent RDK

\section{Keywords}

Onion, Nitrogen,

Phosphorus,

Potassium, Zinc,

Uptake and use

efficiency

Article Info

Accepted:

28 March 2018

Available Online:

10 July 2018

(203.62, 47.33, 236.36 kg ha ${ }^{-1}$ and $347.19 \mathrm{~g} \mathrm{ha}^{-1}$, respectively) over 100 per cent RDK. Among the potassium sources, SOP recorded higher nitrogen and phosphorus, potassium and zinc uptake (171.02 and 40.29, $197.73 \mathrm{~kg} \mathrm{ha}^{-1}$ and $264.02 \mathrm{~g} \mathrm{ha}^{-1}$ respectively) over MOP. The higher nitrogen, phosphorus, potassium and zinc uptake was recorded significantly in application of 50 per cent potassium at transplanting and 50 per cent at 30 DAT $\left(168.52,39.74,195.69 \mathrm{~kg} \mathrm{ha}^{-1}\right.$ and $289.76 \mathrm{~g} \mathrm{ha}^{-1}$ ) over 100 per cent potassium at transplanting $\left(157.90,37.03,187.23 \mathrm{~kg} \mathrm{ha}^{-1}\right.$ and $\left.277.73 \mathrm{~g} \mathrm{ha}^{-1}\right)$. Significantly higher nitrogen and phosphorus use efficiency was recorded in 200 per cent RDK (162.90 and $63.10 \%$, respectively) over 100 per cent RDK. The higher potassium use efficiency on the contrary was recorded in 100 per cent RDK (121.18\%) over 125, 150, 175 and 200 per cent RDK. Among the potassium sources, SOP recorded higher nitrogen, phosphorus and potassium use efficiency of onion plant (136.82, 53.72 and $107.89 \%$, respectively) over MOP. The higher nitrogen, phosphorus and potassium use efficiency was recorded significantly in application of 50 per cent potassium at transplanting and 50 per cent at 30 DAT (134.82, 52.99 and 106.68\%) over 100 per cent potassium at transplanting (126.31, 49.37 and $102.0 \%)$.

\section{Introduction}

Onion (Allium cepa L.) is one of the important commercial bulbous crops cultivated extensively in India and it belongs to the family Alliaceae. It is a most widely grown and popular crop among the Alliums. The primary centre of origin of onion lies in Central Asia (Vavilov, 1951) and the near East and the Mediterranean regions are the secondary centres of origin. It is an ancient crop utilized in medicine, rituals and as a food in Egypt and in India since 600 BC. Onion is mainly used for its flavour and pungency. The 
component which is responsible for pungency in onion is an alkaloid "Allyl propyl disulphide".

The onion is a shallow rooted and potash loving crop, hence it requires fairly higher amount of nutrients including potassium must be maintained in the upper layer ofthe soil. Generally a heavy dose of fertilizer is recommended for onion cultivation (McGillivray, 1961). Like other tuber and root crops, onion is very responsive to potash. Potassium is helpful in many metabolic processes namely production and transport of carbohydrates and sugars, protein synthesis, imparting resistance to pests and diseases, activation of many enzymes, stalk and stem breakage and stress conditions, storage quality, increased bulb size and bulb yield (Pachauri et al., 2005).

Potassium deficiency can bring reduction in production, quality and shelf life of onion. Soils with poor available potassium content usually fail to support satisfactory crop yield (Engels et al., 2012 and Hawkesford et al., 2012). Applying sufficient plant nutrients is needed to sustain the higher production in the face of depleting soil fertility status, continuous cropping and reduced arable land area. Compared with most crops, onion is usually quite susceptible to nutrient deficiency because of their shallow and unbranched root system.

Thus, it requires optimum nutrition which is very well reflected through positive response to the added fertilizers. In addition onions have relatively high demand for soil nutrients, especially $\mathrm{N}, \mathrm{P}$ and $\mathrm{K}$. This has necessitated the application of inorganic fertilizers for maximum growth and yield. However, inappropriate application of inorganic fertilizers may lead to soil acidity or alkalinity. Best quality onion can be produced through the application of balanced nutrition.
Hence, the present investigation is alarmed with the objectives. To study the effect of different methods of application, sources, potassium levels on nutrient uptake and nutrient use efficiency of onion crop.

\section{Materials and Methods}

The present investigation on "Effect of potassium levels, sources and time of application on nutrient uptake and use efficiency of onion var. ArkaKalyan" was carried out at the College of Horticulture, Bagalkot, Karnataka during Kharif season of 2015 and 2016. The details of the materials used and the techniques adopted during the investigation are outlined in this chapter. Bagalkot is situated in the Northern Dry Zone (Zone-3) of Karnataka. The centre is located at $75^{\circ} 42^{\prime}$ East longitude and $16^{\circ} 10^{\prime}$ North latitude with an altitude of $542.00 \mathrm{~m}$ above Mean Sea Level (MSL). The district is grouped under arid and semi-arid region with mean annual rainfall of $517.3 \mathrm{~mm}$ and mean temperature of $32.6^{\circ} \mathrm{C}$. The soil of the experimental site was red sandysoil.

\section{Experimental details}

Treatments: $20(5 \times 2 \times 2)$

Design: Factorial R.B.D

Replications: Three

Season: Kharif

Variety: ArkaKalyan

Spacing: $15 \mathrm{~cm} \times 10 \mathrm{~cm}$

Plot size: $2.1 \mathrm{~m} \times 2.0 \mathrm{~m}$

Fertilizer dose: 125: 75: $125 \mathrm{~kg}$ NPK ha $^{-1}$

Location: Haveli farm, $\mathrm{COH}$, Bagalkot 
Storage period: Three months under ambient condition

\section{Treatment details}

Factor I: Levels of potassium

$100 \%$ RDK + RDNP\&FYM $\left(\mathrm{K}_{1}\right)$

$125 \%$ RDK + RDNP\&FYM $\left(\mathrm{K}_{2}\right)$

$150 \%$ RDK + RDNP\&FYM $\left(\mathrm{K}_{3}\right)$

$175 \%$ RDK + RDNP\&FYM $\left(\mathrm{K}_{4}\right)$

$200 \%$ RDK + RDNP\&FYM $\left(\mathrm{K}_{5}\right)$

Factor II: Sources of potassium: 1. MOP $\left(\mathrm{S}_{1}\right)$, 2. $\operatorname{SOP}\left(\mathrm{S}_{2}\right)$

Factor III: Time of application; $1.100 \% \mathrm{~K}$ at transplanting $\left(\mathrm{T}_{1}\right), 2.50 \% \mathrm{~K}$ at transplanting and $50 \% \quad \mathrm{~K}$ at 30 DAT $\left(\mathrm{T}_{2}\right)$ Note: Recommended dose of NP @ 125:75 kg and FYM@ $@ 30 \mathrm{tha}^{-1}$ was applied commonly to all the treatments and nitrogen was applied $50 \%$ at transplanting and $50 \%$ at 30 days after transplanting.

\section{Total nutrient uptake $\left(\mathrm{kg} \mathrm{ha}^{-1}\right)$}

The plant nutrient (NPK \&ZN) uptake was calculated by multiplying dry weight of leaf and bulb (oven dried at $65^{\circ} \mathrm{C}$ for 48 hours) with nutrient content in plant (leaf and bulb). Further total nutrient uptake was calculated by adding nutrient content of leaf and bulb and was expressed in $\mathrm{kg} \mathrm{ha}^{-1}$.

Dry weight of leaf $x$ Nutrient content in leaf Nutrient uptake by leaf $\left(\mathrm{kg} \mathrm{ha}^{-1}\right)=$

Dry weight of bulb x Nutrient content in bulb Nutrient uptake by bulb $\left(\mathrm{kg} \mathrm{ha}^{-1}\right)=$

100

Total nutrient uptake by plant $\left(\mathrm{kg} \mathrm{ha}^{-1}\right)=$ Nutrient uptake by leaf + Nutrient uptake by bulb
Nutrient content in plant (ppm) $\mathrm{x}$

Yield of dry matter $(\mathrm{kg})$

Nutrient $(\mathrm{Zn})$ uptake $(\mathrm{g}$ ha-1) $=$

1000

\section{Nutrient use efficiency (\%)}

Nutrient (NPK) use efficiency was calculated by using following formula and was expressed in per cent.

Total nutrient uptake Nutrient use efficiency $(\%)=$--------- x 100

Nutrient applied

\section{Results and Discussion}

\section{Nitrogen uptake $\left(\mathrm{kg} \mathrm{ha}^{-1}\right)$}

The data obtained on nutrient uptake and nutrient use efficiency by onion plant in 2015, 2016 and pooled data are presented.

Nitrogen uptake by plant differed significantly due to potassium levels during both the years and in pooled (Table 1). In pooled data the higher nitrogen uptake was recorded significantly in $200 \%$ RDK $\left(203.62 \mathrm{~kg} \mathrm{ha}^{-1}\right)$ over $100 \%, 125 \%, 150 \%$ and $175 \%$ RDK $\left(131.70,143.30,159.66\right.$ and $177.77 \mathrm{~kg} \mathrm{ha}^{-1}$, respectively) and lowest nitrogen uptake was recorded in $100 \%$ RDK. Nitrogen uptake varied significantly by potassium sources during both the years as well as in pooled data. Among the potassium sources, SOP recorded higher nitrogen uptake $\left(171.02 \mathrm{~kg} \mathrm{ha}^{-1}\right)$ over MOP (155.40 kg ha $\left.{ }^{-1}\right)$.

Time of potassium application significantly influenced on nitrogen uptake during 2015 and in pooled data except 2016. In pooled data, higher nitrogen uptake was recorded in application of $50 \%$ potassium at transplanting and $50 \%$ at 30 DAT $\left(168.52 \mathrm{~kg} \mathrm{ha}^{-1}\right)$ over $100 \%$ potassium at transplanting $(157.90 \mathrm{~kg}$ $\mathrm{ha}^{-1}$ ). 
The interaction effect of potassium levels, sources and time of potassium application on nitrogen uptake by plant did not differ significantly during both the years and in pooled data.

\section{Phosphorus uptake (kg ha $\left.{ }^{-1}\right)$}

Phosphorus uptake significantly influenced due to potassium levels during both the years and in pooled (Table 1). In pooled data, higher phosphorus uptake was recorded significantly in $200 \%$ RDK (47.33 kg ha ${ }^{-1}$ ) over $100 \%$, $125 \%, 150 \%$ and $175 \% \operatorname{RDK}(30.97,34.13$, 38.37 and $41.16 \mathrm{~kg} \mathrm{ha}^{-1}$, respectively) and lowest phosphorus uptake was recorded in $100 \%$ RDK.

Phosphorus uptake significantly influenced by potassium sources during both the years as well as in pooled data. Among the potassium sources, SOP recorded higher phosphorus uptake $\left(40.29 \mathrm{~kg} \mathrm{ha}^{-1}\right)$ over MOP $\left(36.49 \mathrm{~kg} \mathrm{ha}^{-}\right.$ $\left.{ }^{1}\right)$.

Time of potassium application significantly influenced on phosphorus uptake during 2015 and in pooled data except 2016.

In pooled data, higher phosphorus uptake was recorded in application of $50 \%$ potassium at transplanting and $50 \%$ at 30 DAT $(39.74 \mathrm{~kg}$ $\mathrm{ha}^{-1}$ ) over $100 \%$ potassium at transplanting (37.03 $\mathrm{kg} \mathrm{ha}^{-1}$ ).

The interaction effect of potassium levels, sources and time of potassium application on phosphorus uptake did not differ significantly during 2016 and in pooled data except 2015. The treatment combination of $\mathrm{K}_{5} \mathrm{~S}_{2} \mathrm{~T}_{2}(200 \%$ RDK, SOP with application of $50 \%$ potassium at transplanting and $50 \%$ at 30 DAT) was recorded significantly higher phosphorus uptake (71.74 $\mathrm{kg} \mathrm{ha}^{-1}$ ) and lowest phosphorus uptake was recorded in $\mathrm{K}_{1} \mathrm{~S}_{1} \mathrm{~T}_{2}\left(28.90 \mathrm{~kg} \mathrm{ha}^{-1}\right)$ in 2015.

\section{Potassium uptake (kg ha $\left.{ }^{-1}\right)$}

Potassium uptake by onion plant significantly influenced due to potassium levels during both the years and in pooled (Table 2). In pooled data, higher potassium uptake was recorded significantly in $200 \%$ RDK (236.36 kg ha $\left.{ }^{-1}\right)$ over $100 \%, 125 \%, 150 \%$ and $175 \%$ RDK (151.48, 171.53, 189.17 and $208.77 \mathrm{~kg} \mathrm{ha}^{-1}$, respectively) and lowest potassium uptake was recorded in $100 \%$ RDK. Potassium uptake varied significantly by potassium sources during both the years as well as in pooled data. Among the potassium sources, SOP recorded higher potassium uptake (197.73 $\left.\mathrm{kg} \mathrm{ha}^{-1}\right)$ over MOP (185.20 kg ha-1).

Time of potassium application significantly influenced in potassium uptake during 2015 and in pooled data except 2016. In pooled data, the higher potassium uptake was recorded in application of $50 \%$ potassium at transplanting and $50 \%$ at 30 DAT $(195.69 \mathrm{~kg}$ $\mathrm{ha}^{-1}$ ) over $100 \%$ potassium at transplanting $\left(187.23 \mathrm{~kg} \mathrm{ha}^{-1}\right)$. The interaction effect of potassium levels, sources and time of potassium application on potassium uptake did not differ significantly during both the years as well as in pooled.

\section{Zinc uptake ( $\left.\mathrm{g} \mathrm{ha}^{-1}\right)$}

Zinc uptake significantly influenced due to potassium levels during both the years and in pooled (Table 2). In pooled data, the higher zinc uptake was recorded significantly in $200 \%$ RDK (347.19 $\mathrm{g} \mathrm{ha}^{-1}$ ) over 100\%, 125\%, $150 \%$ and $175 \%$ RDK $(230.72,251.03$, 285.56 and $304.23 \mathrm{~g} \mathrm{ha}^{-1}$, respectively) and lowest zinc uptake was recorded in $100 \%$ RDK. Zinc uptake varied significantly influenced by potassium sources during both the years as well as in pooled data. Among the potassium sources, SOP recorded higher zinc uptake (303.47 $\mathrm{g} \mathrm{ha}^{-1}$ ) over MOP (264.02 $\mathrm{g} \mathrm{ha}^{-}$ $\left.{ }^{1}\right)$. 
Table.1 Nitrogen and phosphorus uptake $\left(\mathrm{kg} \mathrm{ha}^{-1}\right)$ by onion var. ArkaKalyan as influenced by the soil application of potassium levels, sources and time of application during kharif season

\begin{tabular}{|c|c|c|c|c|c|c|}
\hline \multirow[t]{3}{*}{ Treatment } & \multicolumn{6}{|c|}{ Nutrient uptake $\left(\mathrm{kg} \mathrm{ha}^{-1}\right)$} \\
\hline & \multicolumn{3}{|c|}{ Nitrogen $\left(\mathbf{k g ~ h a}{ }^{-1}\right)$} & \multicolumn{3}{|c|}{ Phosphorus $\left(\mathrm{kg} \mathrm{ha}^{-1}\right)$} \\
\hline & 2015 & 2016 & Pooled & 2015 & 2016 & Pooled \\
\hline \multicolumn{7}{|l|}{ Potassium levels (k) } \\
\hline $\mathrm{K}_{\mathbf{1}}-100 \% \mathrm{RDK}$ & 118.51 & 144.89 & 131.70 & 37.88 & 24.06 & 30.97 \\
\hline $\mathrm{K}_{2}-125 \%$ RDK & 122.24 & 164.37 & 143.30 & 40.10 & 28.17 & 34.13 \\
\hline $\mathrm{K}_{\mathbf{3}^{-150} \% \mathrm{RDK}}$ & 131.71 & 187.61 & 159.66 & 46.80 & 29.93 & 38.37 \\
\hline $\mathrm{K}_{4}-175 \%$ RDK & 142.14 & 213.41 & 177.77 & 46.84 & 35.47 & 41.16 \\
\hline $\mathrm{K}_{5}-200 \% \mathrm{RDK}$ & 172.07 & 235.17 & 203.62 & 56.08 & 38.58 & 47.33 \\
\hline S.Em & 3.99 & 7.12 & 4.54 & 1.90 & 1.25 & 1.18 \\
\hline C.D. $(p=0.05)$ & 11.44 & 20.39 & 13.00 & 5.45 & 3.57 & 3.39 \\
\hline \multicolumn{7}{|l|}{ Potassium sources (S) } \\
\hline$S_{1^{-}}$Muriate of potash (MOP) & 132.81 & 177.99 & 155.40 & 43.00 & 29.99 & 36.49 \\
\hline $\mathbf{S}_{2}$ - Sulphate of potash (SOP) & 141.85 & 200.19 & 171.02 & 48.08 & 32.49 & 40.29 \\
\hline S.Em \pm & 2.53 & 4.50 & 2.87 & 1.20 & 0.79 & 0.75 \\
\hline C.D. $(p=0.05)$ & 7.23 & 12.90 & 8.22 & 3.45 & 2.26 & 2.14 \\
\hline \multicolumn{7}{|l|}{ Time of application (T) } \\
\hline $\mathrm{T}_{1}-100 \% \mathrm{~K}$ at transplanting & 131.30 & 184.49 & 157.90 & 43.27 & 30.79 & 37.03 \\
\hline $\begin{array}{l}\mathrm{T}_{2^{-}} \mathbf{5 0} \% \mathrm{~K} \text { at transplanting } \& \mathbf{5 0} \\
\% \mathrm{~K} \text { at } 30 \mathrm{DAT}\end{array}$ & 143.36 & 193.68 & 168.52 & 47.81 & 31.68 & 39.74 \\
\hline S.Em \pm & 2.53 & 4.50 & 2.87 & 1.20 & 0.79 & 0.75 \\
\hline C.D. $(p=0.05)$ & 7.23 & NS & 8.22 & 3.45 & NS & 2.14 \\
\hline \multicolumn{7}{|l|}{ Interactions } \\
\hline $\mathbf{K}_{1} \mathbf{S}_{1} \mathbf{T}_{1}$ & 100.30 & 126.72 & 113.51 & 31.46 & 20.23 & 25.85 \\
\hline $\mathbf{K}_{1} \mathbf{S}_{1} \mathbf{T}_{2}$ & 95.88 & 131.94 & 113.91 & 28.90 & 20.93 & 24.92 \\
\hline $\mathbf{K}_{1} \mathbf{S}_{2} \mathbf{T}_{1}$ & 113.93 & 158.63 & 136.28 & 34.78 & 27.88 & 31.33 \\
\hline $\mathbf{K}_{1} \mathbf{S}_{2} \mathbf{T}_{2}$ & 163.92 & 162.25 & 163.09 & 56.36 & 27.18 & 41.77 \\
\hline $\mathbf{K}_{2} \mathbf{S}_{1} \mathbf{T}_{1}$ & 129.15 & 153.74 & 141.45 & 41.57 & 27.85 & 34.71 \\
\hline $\mathbf{K}_{2} \mathbf{S}_{1} \mathbf{T}_{2}$ & 125.73 & 158.45 & 142.09 & 40.98 & 31.76 & 36.37 \\
\hline $\mathbf{K}_{2} \mathbf{S}_{2} \mathbf{T}_{1}$ & 103.97 & 171.78 & 137.88 & 35.06 & 26.64 & 30.85 \\
\hline $\mathbf{K}_{2} \mathbf{S}_{2} \mathbf{T}_{2}$ & 130.13 & 173.49 & 151.81 & 42.80 & 26.42 & 34.61 \\
\hline $\mathbf{K}_{3} \mathbf{S}_{1} \mathbf{T}_{1}$ & 141.22 & 166.75 & 153.99 & 51.38 & 31.64 & 41.51 \\
\hline $\mathbf{K}_{3} \mathbf{S}_{1} \mathbf{T}_{2}$ & 135.32 & 204.99 & 170.16 & 43.27 & 27.87 & 35.57 \\
\hline $\mathbf{K}_{3} \mathbf{S}_{2} \mathbf{T}_{1}$ & 128.68 & 190.90 & 159.79 & 49.31 & 31.14 & 40.23 \\
\hline $\mathbf{K}_{3} \mathbf{S}_{2} \mathbf{T}_{2}$ & 121.60 & 187.81 & 154.71 & 43.24 & 29.08 & 36.16 \\
\hline $\mathbf{K}_{4} \mathbf{S}_{1} \mathbf{T}_{1}$ & 124.10 & 188.63 & 156.37 & 41.20 & 30.78 & 35.99 \\
\hline $\mathbf{K}_{4} \mathbf{S}_{1} \mathbf{T}_{2}$ & 144.37 & 203.52 & 173.95 & 47.65 & 35.32 & 41.48 \\
\hline $\mathbf{K}_{4} \mathbf{S}_{2} \mathbf{T}_{1}$ & 141.88 & 228.09 & 184.99 & 50.12 & 34.77 & 42.44 \\
\hline $\mathbf{K}_{4} \mathbf{S}_{2} \mathbf{T}_{2}$ & 158.22 & 233.38 & 195.80 & 48.39 & 41.02 & 44.71 \\
\hline $\mathbf{K}_{5} \mathbf{S}_{1} \mathbf{T}_{1}$ & 163.19 & 219.49 & 191.34 & 48.81 & 36.98 & 42.89 \\
\hline $\mathbf{K}_{5} \mathbf{S}_{1} \mathbf{T}_{2}$ & 168.87 & 225.64 & 197.26 & 54.75 & 36.52 & 45.63 \\
\hline $\mathbf{K}_{5} \mathbf{S}_{2} \mathbf{T}_{1}$ & 166.62 & 240.18 & 203.40 & 49.03 & 40.06 & 44.55 \\
\hline $\mathbf{K}_{5} \mathbf{S}_{2} \mathbf{T}_{2}$ & 189.60 & 255.36 & 222.48 & 71.74 & 40.74 & 56.24 \\
\hline S.Em \pm & 7.99 & 14.24 & 9.08 & 3.81 & 2.49 & 2.37 \\
\hline C.D. $(p=0.05)$ & NS & NS & NS & 10.91 & NS & NS \\
\hline
\end{tabular}

DAT - Days after transplanting, NS-Non significant.

Note: Recommended dose of $\mathrm{N}$ : $\mathrm{P}$ at $125: 75 \mathrm{~kg}$ and farmyard manure $30 \mathrm{t} \mathrm{ha}^{-1}$ was applied commonly to all the treatments and nitrogen was applied $50 \%$ at transplanting and $50 \%$ at 30 DAT. 
Table. 2 Potassium and zinc uptake $\left(\mathrm{kg} \mathrm{ha}^{-1}\right)$ by onion var. ArkaKalyan as influenced by the soil application of potassium levels, sources and time of application during kharif season

\begin{tabular}{|c|c|c|c|c|c|c|}
\hline \multirow[t]{3}{*}{ Treatment } & \multicolumn{6}{|c|}{ Nutrient uptake $\left(\mathrm{kg} \mathrm{ha}^{-1}\right)$} \\
\hline & \multicolumn{3}{|c|}{ Potassium $\left(\mathrm{kg} \mathrm{ha}^{-1}\right)$} & \multicolumn{3}{|c|}{ Zinc $\left(\mathrm{g} \mathrm{ha}^{-1}\right)$} \\
\hline & 2015 & 2016 & Pooled & 2015 & 2016 & Pooled \\
\hline \multicolumn{7}{|l|}{ Potassium levels (k) } \\
\hline $\mathrm{K}_{1}-100 \% \mathrm{RDK}$ & 148.78 & 154.18 & 151.48 & 301.67 & 159.76 & 230.72 \\
\hline $\mathrm{K}_{2}-125 \% \mathrm{RDK}$ & 162.55 & 180.52 & 171.53 & 307.81 & 194.25 & 251.03 \\
\hline $\mathrm{K}_{3}-150 \%$ RDK & 185.77 & 192.58 & 189.17 & 345.93 & 225.18 & 285.56 \\
\hline $\mathrm{K}_{4}-175 \%$ RDK & 189.12 & 228.41 & 208.77 & 348.23 & 260.23 & 304.23 \\
\hline $\mathrm{K}_{5}-200 \% \mathrm{RDK}$ & 224.25 & 248.47 & 236.36 & 403.11 & 291.27 & 347.19 \\
\hline S.Em \pm & 4.82 & 5.87 & 4.43 & 13.90 & 10.76 & 9.26 \\
\hline C.D. $(p=0.05)$ & 13.81 & 16.80 & 12.69 & 39.79 & 30.80 & 26.51 \\
\hline \multicolumn{7}{|l|}{ Potassium sources (S) } \\
\hline$S_{1}-$ Muriate of potash (MOP) & 176.38 & 194.02 & 185.20 & 317.45 & 210.59 & 264.02 \\
\hline $\mathbf{S}_{2}-$ Sulphate of potash (SOP) & 187.81 & 207.64 & 197.73 & 365.25 & 241.70 & 303.47 \\
\hline S.Em \pm & 3.05 & 3.71 & 2.80 & 8.79 & 6.80 & 5.86 \\
\hline C.D. $(p=0.05)$ & 8.73 & 10.62 & 8.03 & 25.16 & 19.48 & 16.77 \\
\hline \multicolumn{7}{|l|}{ Time of application (T) } \\
\hline $\mathrm{T}_{1^{-}} 100 \% \mathrm{~K}$ at transplanting & 176.33 & 198.13 & 187.23 & 331.76 & 223.70 & 277.73 \\
\hline $\begin{array}{l}\mathrm{T}_{2}-50 \% \mathrm{~K} \text { at transplanting } \& 50 \\
\% \mathrm{~K} \text { at } 30 \mathrm{DAT}\end{array}$ & 187.85 & 203.53 & 195.69 & 350.93 & 228.57 & 289.76 \\
\hline S.Em \pm & 3.05 & 3.71 & 2.80 & 8.79 & 6.80 & 5.86 \\
\hline C.D. $(p=0.05)$ & 8.73 & NS & 8.03 & NS & NS & NS \\
\hline \multicolumn{7}{|l|}{ Interactions } \\
\hline $\mathrm{K}_{1} \mathrm{~S}_{1} \mathrm{~T}_{1}$ & 127.89 & 141.23 & 134.56 & 235.96 & 136.27 & 186.12 \\
\hline $\mathrm{K}_{1} \mathrm{~S}_{1} \mathrm{~T}_{2}$ & 139.31 & 156.45 & 147.88 & 257.02 & 141.01 & 199.01 \\
\hline $\mathbf{K}_{1} \mathbf{S}_{2} \mathbf{T}_{1}$ & 146.76 & 162.73 & 154.74 & 296.46 & 193.87 & 245.16 \\
\hline $\mathbf{K}_{1} \mathbf{S}_{2} \mathbf{T}_{2}$ & 181.17 & 156.32 & 168.75 & 417.23 & 167.91 & 292.57 \\
\hline $\mathrm{K}_{2} \mathrm{~S}_{1} \mathrm{~T}_{1}$ & 167.47 & 172.20 & 169.84 & 340.80 & 183.72 & 262.26 \\
\hline $\mathrm{K}_{2} \mathrm{~S}_{1} \mathrm{~T}_{2}$ & 164.60 & 172.35 & 168.48 & 322.12 & 182.86 & 252.49 \\
\hline $\mathrm{K}_{2} \mathrm{~S}_{2} \mathrm{~T}_{1}$ & 153.24 & 188.10 & 170.67 & 264.33 & 202.25 & 233.29 \\
\hline $\mathrm{K}_{2} \mathrm{~S}_{2} \mathrm{~T}_{2}$ & 164.88 & 189.42 & 177.15 & 303.98 & 208.18 & 256.08 \\
\hline $\mathrm{K}_{3} \mathrm{~S}_{1} \mathrm{~T}_{1}$ & 201.61 & 204.50 & 203.06 & 340.29 & 204.25 & 272.27 \\
\hline $\mathrm{K}_{3} \mathrm{~S}_{1} \mathrm{~T}_{2}$ & 172.74 & 182.54 & 177.64 & 330.54 & 244.73 & 287.63 \\
\hline $\mathrm{K}_{3} \mathrm{~S}_{2} \mathrm{~T}_{1}$ & 183.15 & 187.95 & 185.55 & 413.86 & 242.60 & 328.23 \\
\hline $\mathrm{K}_{3} \mathrm{~S}_{2} \mathrm{~T}_{2}$ & 185.57 & 195.34 & 190.45 & 299.05 & 209.16 & 254.10 \\
\hline $\mathrm{K}_{4} \mathrm{~S}_{1} \mathrm{~T}_{1}$ & 159.25 & 196.92 & 178.09 & 307.34 & 236.22 & 271.78 \\
\hline $\mathrm{K}_{4} \mathrm{~S}_{1} \mathrm{~T}_{2}$ & 194.25 & 245.15 & 219.70 & 322.91 & 219.63 & 271.27 \\
\hline $\mathrm{K}_{4} \mathrm{~S}_{2} \mathrm{~T}_{1}$ & 200.88 & 225.34 & 213.11 & 400.59 & 283.96 & 342.27 \\
\hline $\mathrm{K}_{4} \mathrm{~S}_{2} \mathrm{~T}_{2}$ & 202.11 & 246.23 & 224.17 & 362.10 & 301.13 & 331.61 \\
\hline $\mathrm{K}_{5} \mathrm{~S}_{1} \mathrm{~T}_{1}$ & 216.53 & 239.86 & 228.20 & 339.46 & 261.46 & 300.46 \\
\hline $\mathrm{K}_{5} \mathrm{~S}_{1} \mathrm{~T}_{2}$ & 220.13 & 229.00 & 224.57 & 378.04 & 295.73 & 336.88 \\
\hline $\mathrm{K}_{5} \mathrm{~S}_{2} \mathrm{~T}_{1}$ & 206.54 & 262.50 & 234.52 & 378.52 & 292.48 & 335.50 \\
\hline $\mathrm{K}_{5} \mathrm{~S}_{2} \mathrm{~T}_{2}$ & 253.79 & 262.52 & 258.15 & 516.40 & 315.42 & 415.91 \\
\hline S.Em \pm & 9.65 & 11.73 & 8.87 & 27.79 & 21.52 & 18.52 \\
\hline C.D. $(p=0.05)$ & NS & NS & NS & 79.57 & NS & NS \\
\hline
\end{tabular}

DAT - Days after transplanting, NS-Non significant.

Note: Recommended dose of $\mathrm{N}: \mathrm{P}$ at $125: 75 \mathrm{~kg}$ and farmyard manure $30 \mathrm{t} \mathrm{ha}^{-1}$ was applied commonly to all the treatments and nitrogen was applied $50 \%$ at transplanting and $50 \%$ at 30 DAT. 
Table.3 Nitrogen, phosphorus and potassium use efficiency (\%) of onion var. ArkaKalyan as influenced by the soil application of potassium levels, sources and time of application during kharif season

\begin{tabular}{|c|c|c|c|c|c|c|c|c|c|}
\hline \multirow[t]{3}{*}{ Treatment } & \multicolumn{9}{|c|}{ Nutrient use efficiency (\%) } \\
\hline & \multicolumn{3}{|c|}{ Nitrogen (\%) } & \multicolumn{3}{|c|}{ Phosphorus (\%) } & \multicolumn{3}{|c|}{ Potassium (\%) } \\
\hline & 2015 & 2016 & Pooled & 2015 & 2016 & Pooled & 2015 & 2016 & Pooled \\
\hline \multicolumn{10}{|l|}{ Potassium levels (k) } \\
\hline $\mathrm{K}_{1}-100 \% \mathrm{RDK}$ & 94.81 & 115.91 & 105.36 & 50.50 & 32.08 & 41.29 & 119.02 & 123.35 & 121.18 \\
\hline $\mathrm{K}_{2}-125 \%$ RDK & 97.79 & 131.49 & 114.64 & 53.47 & 37.55 & 45.51 & 104.03 & 115.53 & 109.78 \\
\hline $\mathrm{K}_{3}-150 \%$ RDK & 105.36 & 150.09 & 127.73 & 62.40 & 39.91 & 51.15 & 99.08 & 102.71 & 100.89 \\
\hline $\mathrm{K}_{4}-175 \%$ RDK & 113.71 & 170.73 & 142.22 & 62.45 & 47.30 & 54.87 & $86 . .46$ & 104.42 & 95.44 \\
\hline $\mathrm{K}_{5}-200 \%$ RDK & 137.66 & 188.14 & 162.90 & 74.77 & 51.43 & 63.10 & 89.70 & 99.39 & 94.54 \\
\hline S.Em \pm & 3.20 & 5.70 & 3.63 & 2.54 & 1.66 & 1.58 & 2.55 & 2.90 & 2.18 \\
\hline C.D. $(p=0.05)$ & 9.15 & 16.31 & 10.40 & 7.27 & 4.76 & 4.52 & 7.30 & 8.29 & 6.25 \\
\hline \multicolumn{10}{|l|}{ Potassium sources (S) } \\
\hline$S_{1-}$ Muriate of potash (MOP) & 106.25 & 142.39 & 124.32 & 57.33 & 39.98 & 48.66 & 96.22 & 105.47 & 100.85 \\
\hline $\mathrm{S}_{2^{-}}$Sulphate of potash (SOP) & 113.48 & 160.15 & 136.82 & 64.11 & 43.32 & 53.72 & 103.09 & 112.69 & 107.89 \\
\hline S.Em \pm & 2.02 & 3.60 & 2.30 & 1.61 & 1.05 & 1.00 & 1.61 & 1.83 & 1.38 \\
\hline C.D. $(p=0.05)$ & 5.79 & 10.32 & 6.58 & 4.60 & 3.01 & 2.86 & 4.62 & 5.24 & 3.95 \\
\hline \multicolumn{10}{|l|}{ Time of application (T) } \\
\hline $\mathrm{T}_{1}-100 \% \mathrm{~K}$ at transplanting & 105.04 & 147.59 & 126.31 & 57.69 & 41.06 & 49.37 & 96.40 & 107.70 & 102.05 \\
\hline $\begin{array}{l}\mathrm{T}_{2^{-}} 50 \% \mathrm{~K} \text { at transplanting } \& \\
50 \% \mathrm{~K} \text { at } 30 \mathrm{DAT}\end{array}$ & 114.69 & 154.95 & 134.82 & 63.74 & 42.24 & 52.99 & 102.91 & 110.45 & 106.68 \\
\hline S.Em \pm & 2.02 & 3.60 & 2.30 & 1.61 & 1.05 & 1.00 & 1.61 & 1.83 & 1.38 \\
\hline C.D. $(p=0.05)$ & 5.79 & NS & 6.58 & 4.60 & NS & 2.86 & 4.62 & NS & 3.95 \\
\hline \multicolumn{10}{|l|}{ Interactions } \\
\hline $\mathbf{K}_{1} \mathbf{S}_{1} \mathbf{T}_{1}$ & 80.24 & 101.38 & 90.81 & 41.95 & 26.97 & 34.46 & 102.31 & 112.98 & 107.65 \\
\hline $\mathbf{K}_{1} \mathbf{S}_{1} \mathbf{T}_{2}$ & 76.71 & 105.55 & 91.13 & 38.54 & 27.91 & 33.22 & 111.45 & 125.16 & 118.30 \\
\hline $\mathbf{K}_{1} \mathbf{S}_{2} \mathbf{T}_{1}$ & 91.15 & 126.91 & 109.03 & 46.37 & 37.18 & 41.77 & 117.41 & 130.18 & 123.79 \\
\hline $\mathbf{K}_{1} \mathbf{S}_{2} \mathbf{T}_{2}$ & 131.14 & 129.80 & 130.47 & 75.15 & 36.25 & 55.70 & 144.93 & 125.06 & 135.00 \\
\hline $\mathrm{K}_{2} \mathrm{~S}_{1} \mathrm{~T}_{1}$ & 103.32 & 123.00 & 113.16 & 55.43 & 37.13 & 46.28 & 107.18 & 110.21 & 108.70 \\
\hline $\mathbf{K}_{2} \mathbf{S}_{1} \mathbf{T}_{2}$ & 100.58 & 126.76 & 113.67 & 54.64 & 42.35 & 48.50 & 105.34 & 110.31 & 107.82 \\
\hline $\mathbf{K}_{2} \mathbf{S}_{2} \mathbf{T}_{1}$ & 83.18 & 137.42 & 110.30 & 46.75 & 35.51 & 41.13 & 98.07 & 120.38 & 109.23 \\
\hline $\mathbf{K}_{2} \mathbf{S}_{2} \mathbf{T}_{2}$ & 104.10 & 138.79 & 121.45 & 57.07 & 35.22 & 46.14 & 105.52 & 121.23 & 113.38 \\
\hline $\mathrm{K}_{3} \mathbf{S}_{1} \mathbf{T}_{1}$ & 112.98 & 133.40 & 123.19 & 68.50 & 42.18 & 55.34 & 107.53 & 109.07 & 108.30 \\
\hline $\mathrm{K}_{3} \mathbf{S}_{1} \mathbf{T}_{2}$ & 108.26 & 163.99 & 136.13 & 57.70 & 37.16 & 47.43 & 92.13 & 97.35 & 94.74 \\
\hline $\mathrm{K}_{3} \mathbf{S}_{2} \mathbf{T}_{1}$ & 102.94 & 152.72 & 127.83 & 65.75 & 41.52 & 53.63 & 97.68 & 100.24 & 98.96 \\
\hline $\mathrm{K}_{3} \mathrm{~S}_{2} \mathbf{T}_{2}$ & 97.28 & 150.25 & 123.76 & 57.65 & 38.77 & 48.21 & 98.97 & 104.18 & 101.57 \\
\hline $\mathrm{K}_{4} \mathbf{S}_{1} \mathbf{T}_{1}$ & 99.28 & 150.90 & 125.09 & 54.94 & 41.04 & 47.99 & 72.80 & 90.02 & 81.41 \\
\hline $\mathrm{K}_{4} \mathrm{~S}_{1} \mathrm{~T}_{2}$ & 115.50 & 162.82 & 139.16 & 63.53 & 47.10 & 55.31 & 88.80 & 112.07 & 100.43 \\
\hline $\mathrm{K}_{4} \mathbf{S}_{2} \mathbf{T}_{1}$ & 113.51 & 182.48 & 147.99 & 66.82 & 46.36 & 56.59 & 91.83 & 103.01 & 97.42 \\
\hline $\mathbf{K}_{4} \mathbf{S}_{2} \mathbf{T}_{2}$ & 126.57 & 186.70 & 156.64 & 64.52 & 54.70 & 59.61 & 92.39 & 112.56 & 102.48 \\
\hline $\mathbf{K}_{5} \mathbf{S}_{1} \mathbf{T}_{1}$ & 130.55 & 175.59 & 153.07 & 65.07 & 49.31 & 57.19 & 86.61 & 95.94 & 91.28 \\
\hline $\mathbf{K}_{5} \mathbf{S}_{1} \mathbf{T}_{2}$ & 135.10 & 180.52 & 157.81 & 73.00 & 48.69 & 60.84 & 88.05 & 91.60 & 89.83 \\
\hline $\mathrm{K}_{5} \mathbf{S}_{2} \mathbf{T}_{1}$ & 133.29 & 192.14 & 162.72 & 65.37 & 53.42 & 59.39 & 82.62 & 105.00 & 93.81 \\
\hline $\mathbf{K}_{5} \mathbf{S}_{2} \mathbf{T}_{2}$ & 151.68 & 204.29 & 177.98 & 95.66 & 54.32 & 74.99 & 101.52 & 105.01 & 103.26 \\
\hline S.Em \pm & 6.39 & 11.40 & 7.27 & 5.08 & 3.32 & 3.16 & 5.10 & 5.79 & 4.37 \\
\hline C.D. $(p=0.05)$ & NS & NS & NS & 14.54 & NS & NS & NS & NS & NS \\
\hline
\end{tabular}

DAT - Days after transplanting, NS-Non significant.

Note: Recommended dose of $\mathrm{N}$ : $\mathrm{P}$ at $125: 75 \mathrm{~kg}$ and farmyard manure $30 \mathrm{t} \mathrm{ha}^{-1}$ was applied commonly to all the treatments and nitrogen was applied $50 \%$ at transplanting and $50 \%$ at 30 DAT. 
Time of potassium application did not differ significantly in zinc uptake during both the years and in pooled data. In pooled data, higher zinc uptake was recorded in application of $50 \%$ potassium at transplanting and $50 \%$ at 30 DAT (289.76 $\mathrm{g} \mathrm{ha}^{-1}$ ) over $100 \%$ potassium at transplanting $(277.73 \mathrm{~g}$ ha $\left.{ }^{1}\right)$.

The interaction effect of potassium levels, sources and time of potassium application on zinc uptake did not differ significantly during 2016 and in pooled except 2015. The treatment combination of $\mathrm{K}_{5} \mathrm{~S}_{2} \mathrm{~T}_{2} \quad(200 \%$ RDK, SOP with application of $50 \%$ potassium at transplanting and $50 \%$ at 30 DAT) was recorded significantly higher zinc uptake (516.40 $\mathrm{g} \mathrm{ha}^{-1}$ ) and lowest zinc uptake by onion plant was recorded in $\mathrm{K}_{1} \mathrm{~S}_{1} \mathrm{~T}_{1}$ (235.96 $\left.\mathrm{g} \mathrm{ha}^{-1}\right)$.

\section{Nitrogen use efficiency $(\%)$}

Nitrogen use efficiency of onion plant significantly influenced due to potassium levels during both the years as well as in pooled (Table 3 ). In pooled data, the higher nitrogen use efficiency was recorded significantly in $200 \%$ RDK (162.90\%) over $100 \%, 125 \%, 150 \%$ and $175 \%$ RDK (105.36, $114.64,127.73$ and $142.22 \%$, respectively) and minimum nitrogen use efficiency was recorded in $100 \%$ RDK.

Nitrogen use efficiency significantly influenced by potassium sources during both the years as well as in pooled data. Among the potassium sources, SOP recorded higher nitrogen use efficiency (136.82\%) over MOP (124.32\%).

Time of potassium application differ significantly in nitrogen use efficiency during 2015 and in pooled data except 2016. In pooled data, the higher nitrogen use efficiency was recorded significantly in application of
$50 \%$ potassium at transplanting and $50 \%$ at 30 DAT $(134.82 \%)$ over $100 \%$ potassium at transplanting (126.31\%).

The interaction effect of potassium levels, sources and time of potassium application on nitrogen use efficiency of onion plant did not differ significantly during both the years and in pooled.

\section{Phosphorus use efficiency (\%)}

Phosphorus use efficiency significantly influenced due to potassium levels during both the years as well as in pooled (Table 3 ). In pooled data, the higher phosphorus use efficiency was recorded significantly in $200 \%$ RDK $(63.10 \%)$ over $100 \%, 125 \%, 150 \%$ and $175 \%$ RDK (41.29, 45.51, 51.15 and $54.87 \%$, respectively) and minimum phosphorus use efficiency was recorded in $100 \%$ RDK.

Phosphorus use efficiency varied significantly by potassium sources during both the years as well as in pooled data. Among the potassium sources, SOP recorded higher phosphorus use efficiency $(53.72 \%)$ over MOP (48.66\%).

Time of potassium application differ significantly in phosphorus use efficiency during 2015 and in pooled data except 2016. In pooled data, the higher phosphorus use efficiency was recorded significantly in application of $50 \%$ potassium at transplanting and $50 \%$ at 30 DAT $(52.99 \%)$ over $100 \%$ potassium at transplanting $(49.37 \%)$.

The interaction effect of potassium levels, sources and time of potassium application on phosphorus use efficiency did not differ significantly during 2016 and in pooled except 2015. The treatment combination of $\mathrm{K}_{5} \mathrm{~S}_{2} \mathrm{~T}_{2}(200 \%$ RDK, SOP with application of $50 \%$ potassium at transplanting and $50 \%$ at 30 DAT) was recorded significantly higher phosphorus use efficiency of onion plant 
$(95.66 \%)$ and minimum was recorded in $\mathrm{K}_{1} \mathrm{~S}_{1} \mathrm{~T}_{2}(38.54 \%)$.

\section{Potassium use efficiency (\%)}

Potassium use efficiency significantly influenced due to potassium levels during both the years as well as in pooled (Table 3 ). In pooled data, the higher potassium use efficiency was recorded significantly in $100 \%$ RDK (121.18\%) over $125 \%, 150 \% 175 \%$ and $200 \%$ RDK (109.78, 100.89, 95.44 and $94.54 \%$, respectively) and minimum potassium use efficiency was recorded in $200 \%$ RDK.

Potassium use efficiency significantly influenced by potassium sources during both the years as well as in pooled data. Among the potassium sources, SOP recorded higher phosphorus use efficiency $(107.89 \%)$ over MOP $(100.85 \%)$.

Time of potassium application differed significantly in potassium use efficiency during 2015 and in pooled data except 2016. In pooled data, the higher potassium use efficiency was recorded significantly in application of $50 \%$ potassium at transplanting and $50 \%$ at 30 DAT $(106.68 \%)$ over $100 \%$ potassium at transplanting $(102.05 \%)$.

The interaction effect of potassium levels, sources and time of potassium application on potassium use efficiency did not differ significantly during both the years as well as in pooled.

The uptake of NPK and $\mathrm{Zn}$ increased with levels of potassium. In general, the increased uptake of nitrogen, phosphorus, potassium and zinc recorded in 200 per cent RDK (203.62, 47.33, $236.36 \mathrm{~kg} \mathrm{ha}^{-1}$ and $347.19 \mathrm{~g}$ ha $^{-1}$, respectively) over 100 per cent RDK. This increased uptake of nutrients could be attributed to increased dry matter production under increased potassium levels. Watson (1963) has attributed such a higher nutritional uptake mainly by greater expansion of root system caused by increased supply of photosynthates. The higher uptake of nutrients with addition of potassium might be attributed to increased vigour of crop growth with enhanced nutrient utilization and translocation into the plant resulting in the enhancement of bulb yield. These results are in conformity with the earlier findings of Hariyappa et al., (2011), Shafeek et al., (2013), Deshpande et al., (2013) and Poornima et al., (2015).

Nitrogen, phosphorus and potassium use efficiency was significantly influenced due to potassium levels. Significantly higher nitrogen and phosphorus use efficiency was recorded in 200 per cent RDK (162.90 and $63.10 \%$, respectively) over 100 per cent RDK. Similarly potassium use efficiency influenced due to potassium levels. The higher potassium use efficiency on the contrary was recorded in 100 per cent RDK (121.18\%) over 125,150 , 175 and 200 per cent RDK (109.78, 100.89, 95.44 and $94.54 \%$, respectively). The nutrient use efficiency of nitrogen and phosphorus increased with increased levels of potassium. However, inverse relationship was observed between potassium use efficiency and its application rate. Increasing levels of potassium there is a imbalance of nutrients mainly inturns nitrogen and phosphorus which resulted in the low use efficiency of potassium applied. In the present study the higher residual potash in the soil with the lower level of available soil phosphorus at 200 per cent RDK clearly indicates the need of balanced nutrient application to enhance the potassium use efficiency. The potassium use efficiency was found decreasing in the 100 per cent-200 per cent of potassium application. The variation in nutrient use efficiency of applied nutrient could be attributed to the combined effect of all the 
nutrients on the whole plant system (Assefa $e t$ al., 2015). There are many reports of higher nutrient use efficiencies through balanced fertilizer application (Nasreen et al., 2007a). Similar results on nutrient use efficiency of onion are being reported by Anwar et al., (2001) and Majumdar et al., (2003).

Nitrogen, phosphorus, potassium and zinc uptake by plant differed significantly by potassium sources. Among the potassium sources, SOP recorded higher nitrogen and phosphorus, potassium and zinc uptake (171.02 and 40.29, $197.73 \mathrm{~kg} \mathrm{ha}^{-1}$ and 264.02 $\mathrm{g} \mathrm{ha}^{-1}$ respectively) over MOP. This increased uptake of nutrients due to application of sulphate of potash could be attributed to increased uptake by different plant parts and increased dry matter production under supply of nutrients especially potassium and sulphur. Watson (1963) has attributed such a higher nutritional uptake mainly to greater expansion of root system caused by increased supply of photosynthetic production. The higher uptake of nutrients with addition of potassium might be attributed to increased vigour of crop growth with enhanced nutrient utilization and translocation into the plant resulting in the enhancement of bulb yield as reported by Desuki et al., (2006). These results are in conformity with the earlier findings of Girigowda et al., (2005), Verma and Singh (2012), shafeek et al., (2013) and Deshpande et al., (2013),

Nitrogen, phosphorus and potassium use efficiency was significantly influenced by potassium sources. Among the potassium sources, SOP recorded higher nitrogen, phosphorus and potassium use efficiency of onion plant (136.82, 53.72 and $107.89 \%$, respectively) over MOP (124.32, 48.66 and $100.85 \%$, respectively). In the present investigation among the potassium sources, SOP recorded the higher nutrient use efficiency with respect to nitrogen, phosphorus and potassium due to application of sulphate of potash as compared to muriate of potash. This may be because of application potassium sources along with nitrogen and phosphorus which are responsible for increasing nutrient uptake by the crop. Uptake of nutrients increased with increasing availability of nutrient and also with nutrient use efficiency. The variation in nutrient use efficiency of applied nutrient could be attributed to the combined effect of all the nutrients on the whole plant system (Assefa $e t$ al., 2015). Similar results on nutrient use efficiency of onion are reported by Anwar et al., (2001), Majumdar et al., (2003) and Desuki et al., (2006).

The higher nitrogen, phosphorus, potassium and zinc uptake was recorded in application of 50 per cent potassium at transplanting and 50 per cent at 30 DAT $(168.52,39.74,195.69$ $\mathrm{kg} \mathrm{ha}^{-1}$ and $289.76 \mathrm{~g} \mathrm{ha}^{-1}$ ) over 100 per cent potassium at transplanting $(157.90,37.03$, $187.23 \mathrm{~kg} \mathrm{ha}^{-1}$ and $\left.277.73 \mathrm{~g} \mathrm{ha}^{-1}\right)$. Time of potassium application differ significantly in nutrient use efficiency of onion plant. The higher nitrogen, phosphorus and potassium use efficiency was recorded significantly in application of 50 per cent potassium at transplanting and 50 per cent at 30 DAT (134.82, 52.99 and $106.68 \%$ ) over 100 per cent potassium at transplanting $(126.31,49.37$ and $102.0 \%)$.

The result indicated that the nutrient uptake and nutrient use efficiency increased with application 50 per cent potassium at transplanting and 50 per cent $\mathrm{K}$ at $30 \mathrm{DAT}$ with recommended dose of nitrogen and phosphorus. The higher amount of nutrient in plant leaves and bulb may be due to higher nutrient uptake by onion plant which resulted in vigorous plant growth. If the plants shows more growth then it is inevitable that plant takes more nutrients from the soil. This might be attributed to increased dry matter 
production. It is known that potash helps in root development and increases the efficiency of leaf in manufacturing sugars and starch. Though split application, potash is likely to be utilized by the crop more efficiently. Significantly improvement in nutrient uptake and use efficiency by onion plant due to split application of potash. These findings are in agreement with the results of Singh and Verma (2001) and Islam et al., (2008).

The interaction effect of potassium levels, sources and time of potassium application on nitrogen, phosphorus, potassium and zinc uptake. The treatment combination of $\mathrm{K}_{5} \mathrm{~S}_{2} \mathrm{~T}_{2}$ (200\% RDK, SOP with application of 50 per cent potassium at transplanting and 50 per cent at 30 DAT) was recorded higher nitrogen, phosphorus, potassium and zinc uptake by onion plant $(222.48,71.74,258.15$ $\mathrm{kg} \mathrm{ha}^{-1}$ and $\left.516.40 \mathrm{~g} \mathrm{ha}^{-1}\right)$. The interaction effect of potassium levels, sources and time of potassium application on nitrogen and phosphorus use efficiency. The treatment combination of $\mathrm{K}_{5} \mathrm{~S}_{2} \mathrm{~T}_{2}(200 \%$ RDK, SOP with application of 50 per cent potassium at transplanting and 50 per cent at 30 DAT) was recorded maximum nitrogen use efficiency $(177.98,95.66 \%)$. The treatment combination of $\mathrm{K}_{1} \mathrm{~S}_{2} \mathrm{~T}_{2}$ (100 per cent RDK, SOP with application of 50 per cent potassium at transplanting and 50 per cent at 30 DAT) was recorded higher potassium use efficiency $(135.00 \%)$. Increased nutrient uptake was also observed due to the interaction effects of potassium levels, sources and time of application. The increased nutrient uptake could be attributed to increased dry matter production under supply of potassium levels, sources especially potassium and sulphur present in sulphate of potash and split application of potash with nitrogen during the crop growth period. The higher nutrient uptake might be attributed to increased vigour of crop growth with enhanced nutrient utilization and translocation into the plant resulting in the higher yield ultimately increased the nutrient use efficiency by the crop. These results are in conformity with the finding of Desuki et al., (2006) and ElBassiony (2006).

\section{References}

Anwar, M. N., Sarker, J. U., Rahman, M., Islam, M. A. and Begum, M., 2001. Response of onion to nitrogen, phosphorus, potassium, sulphur and zinc. Bangladesh J. Environ. Sci.,7: 6872.

Assefa, A. G., Mesgina, S. H. and Abrha, Y. W., 2015. Effect of inorganic and organic fertilizers on the growth and yield of garlic crop in Northern Ethiopia. J. Agril Sci., 7 (4): 191-197.

Deshpande, A. N., Dage, A. R., Bhalerao, V. P. and Bansal, S. K., 2013. Potassium nutrition for improving yield and quality of onion. Int. Potash Institute, 36: 214-222.

Desuki, M. M., Abdel-Mouty and Ali, A. H., 2006. Response of onion plants to additional dose of potassium application. J. Appl. Sci. Res., 2 (9): 592597.

El-Bassiony, A. M., 2006. Effect of potassium fertilization on growth, yield and quality of onion plants. J. Appl. Sci. Res.,2 (10): 780-785.

Engels, C., Kirkby, E. A. and White, P., 2012. Mineral nutrition, yield and source sink relationships. In: Marschner, P. (ed.) Marschner's mineral nutrition of higher plants, $3^{\text {rd }}$ edition. Elsevier Ltd. pp. 8597.

Girigowda, J. R., Narasegowda, N. C. and Krishna, H. C., 2005. Effect of fertilizer levels on uptake of primary nutrients and bulb yield of onion hybrids. Mysore J. Agric. Sci. 39 (4): 557-560.

Hariyappa, N., Halawar, A. B. and Hebsur, N.S., 2011. Influence of potassium and 
sulphur levels on uptake of nutrient in onion (Allium cepa L.). Asian J. Soil Sci., 6 (2): 185-188.

Hawkesford, M., Horst, W., Kichey, T., Lambers, H., Schjoerring, J., Skrumsager, M. I. and White, P., 2012. Functions of macronutrients. In: Marshner, P. (ed.) Marschner's Mineral Nutrition of Higher Plants, $3^{\text {rd }}$ edition. Elsevier Ltd., pp. 135-151.

Islam, M. A., Sharnsuddoha, A. T. M, Bhuiyan M S. I. and Hasanmzaman, M., 2008. Response of summer onion to potash and its application methods. American. Eurasian J. Agron., 1 (1): 10-15.

Majumdar, B., Venkatesh, M. S., Kailash, K. and Patiram, K., 2003. Response of garlic to phosphorus and sulphur application in acid alfisol of Meghalaya. J. Spices and Aromatic Crops,12 (2): 183-186.

McGillivary, J. H., 1961. Vegetable production. Mc Grew Hill Book Co. Inc. New York, pp.169-273.

Nasreen, S., Haque M. M., Hossain, M. A. and Farid, A. T. M., 2007a. Nutrient uptake and yield of onion as influenced by nitrogen and sulphur fertilization. Bangladesh J. Agric. Res.,32 (3): 413420.
Pachauri, S. P., Singh, V. and Pachauri, C. P., 2005. Effect of FYM, nitrogen and potassium on growth, yield and quality of onion. Ann. Pl. Soil Res., 7 (1):54-56.

Poornima, K. S., Mamatha, N. and Ramesh, H. S., 2015. Effect of potassium and sulphur on quality parameters of onion and chilli intercrops in a vertisol. $A d v$. Res. J. Crop improvement, 6 (2):166169.

Shafeek, M. R., Nagwa, M. K., Hassan, S., Singer, M. and Nadia, H. M., Greadly, E. L., 2013. Effect of potassium fertilizer and foliar spraying with Etherel on plant development, yield and bulb quality of onion plants (Allium cepaL). J. Appl. Sci. Res., 9 (2): 11401146.

Singh, S. P. and Verma, A. B., 2001. Response of onion (Allium cepa) to potassium application. Indian J. Agron., 46 (1): 182-185.

Vavilov, N. I., 1951. Origin, variation, immunity and breeding of cultivated plants. J. Chronical Bot., 13 (1/6): 1364.

Watson, D. J., 1963. In the growth of potato. Eds. Ivins, J.D. and Mirthorpe, F.L., Butter Worths, London, pp. 233-247.

\section{How to cite this article:}

Kumara B. R., C. P. Mansur, Shankar Meti, S. L. Jagadeesh, Girish Chander, S. P. Wani, R. K. Mesta, D. Satish, T. B. Allolli and Sanjeev Reddy. 2018. Potassium Levels, Sources and Time of Application on Nutrient Uptake and Nutrient Use Efficiency of Onion (Allium cepa L.). Int.J.Curr.Microbiol.App.Sci. 7(07): 4214-4225. doi: https://doi.org/10.20546/ijcmas.2018.707.493 